\title{
Validity of the Law of Mass Action in Three-Dimensional Coagulation Processes
}

\author{
Anton A. Winkler and Erwin Frey \\ Arnold Sommerfeld Center for Theoretical Physics and Center for NanoScience, Department of Physics, Ludwig-Maximilians- \\ Universität München, Theresienstraße 37, 80333 München, Germany
}

(Received 24 June 2011; published 5 March 2012)

\begin{abstract}
Diffusion-limited reactions are studied in detail on the classical coalescing process. We demonstrate how, with the aid of a recent renormalization group approach, fluctuations can be integrated systematically. We thereby obtain an exact relation between the microscopic physics (lattice structure and particle shape and size) and the macroscopic decay rate in the law of mass action. Moreover, we find a strong violation of the law of mass action. The corresponding term in the kinetic equations originates in longwavelength fluctuations and is a universal function of the macroscopic decay rate.
\end{abstract}

PACS numbers: 82.20.- w, 05.10.Cc, 05.40.-a, 64.60.Ht

The law of mass action (LMA) is the fundamental law in chemical reaction kinetics. It states that the rate of an elementary reaction is proportional to the product of the concentrations of the participating molecules. In a seminal article that helped lay the foundations of a stochastic theory of chemical reaction kinetics, Smoluchowski provided a framework for the calculation of macroscopic decay rates and supported the validity of the LMA for three-dimensional systems [1,2]. In the 1980s much effort was put into studying low-dimensional systems, where it was found that strong correlations can lead to deviations from the LMA [3-7]. This anomalous behavior was observed, in particular, for the classical problem of coalescence, $A+A \rightarrow A$, where diffusing particles clot upon contact with a rate $\lambda$. By an approach designed for one dimension, one could even obtain exact solutions [8]. This was complemented by results of the perturbative renormalization group at and below the critical dimension $d_{c}=2$ $[9,10]$. In contrast to this progress in low dimensions, advances for three-dimensional coagulation systems have remained largely elusive. In the experimental analysis, the LMA is still the "gold standard" [11-13]. Indeed, it has obtained further support by field theoretic analysis proving the validity of Smoluchowski's heuristic arguments for asymptotically long times and low densities $[14,15]$. In this regime the density $\rho$ obeys the LMA rate equation $\partial_{t} \rho=-\mu \rho^{2}$, with a macroscopic decay rate $\mu$ which is a function of the microscopic rate $\lambda$ and of the size and shape of the particles.

In this Letter, we employ a nonperturbative renormalization group approach [16-19] to study coagulation processes in three spatial dimensions. We find that the density obeys an equation of motion $\partial_{t} \rho=-F(\rho)$, where the nonequilibrium "force" $F$ is derived from a nonequilibrium analog of a thermodynamic potential. It is obtained upon employing a nonperturbative renormalization group procedure, which successively integrates fluctuations starting at the microscopic scale. This provides a profound understanding of the intimate connection between the macroscopic description and the microscopic properties of the kinetic process. For low densities we recover the result from the law of mass action: $F(\rho) \approx \mu \rho^{2}$. An exact flow equation is derived which connects the microscopic rate $\lambda$ to the (nonuniversal) macroscopic rate $\mu$. Moreover, in contrast to previous work, the renormalization group approach enables us to explicitly incorporate both the effect of lattice structure and of shape and size of the particles. Last, we can calculate the nonequilibrium force $F(\rho)$ beyond the low-density limit. We find that longwavelength fluctuations give rise to a nonanalytic term of the form $c(\mu) \rho^{5 / 2}$. Remarkably, $c(\mu)$ is a simple, universal function of the macroscopic rate $\mu$. It depends on the microscopic features of the lattice and the particles only indirectly through its argument $\mu$.

The coagulation process can be recast in terms of a field theory, an approach devised by several authors [20-22]. It has proven a powerful and versatile framework for reaction-diffusion processes in the past and also forms the basis of our nonperturbative renormalization group (RG) calculations. (For a simple example of the procedure, see [23].) The stochastic dynamics corresponding to the reaction scheme $A+A \rightarrow A$ on a three-dimensional lattice is first mapped to its master equation and then translated to the action

$$
\begin{aligned}
S[\bar{\psi}, \psi]= & S_{\lambda}[\bar{\psi}, \psi]+S_{\epsilon}[\bar{\psi}, \psi] \\
& +\int d t \sum_{\mathbf{x}} \bar{\psi}(\mathbf{x}, t) \partial_{t} \psi(\mathbf{x}, t),
\end{aligned}
$$

where the fields $\bar{\psi}$ and $\psi$ are related to creation and annihilation of particles, respectively. We wish to study particles that may extend over several lattice sites. This is achieved by introducing a reaction kernel $\lambda(\mathbf{y}-\mathbf{x})$ defining the rate at which a particle at site $\mathbf{x}$ annihilates another particle at site $\mathbf{y}$. The coagulation term $S_{\lambda}[\bar{\psi}, \psi]$ then reads

$$
\int d t \sum_{\mathbf{x}, \mathbf{y}} \lambda(\mathbf{y}-\mathbf{x})[\bar{\psi}(\mathbf{y}, t)+1] \bar{\psi}(\mathbf{x}, t) \psi(\mathbf{y}, t) \psi(\mathbf{x}, t) .
$$


While this determines the shape and size of the particles, the lattice structure is encoded in the diffusion term. In Fourier space it is of the form

$$
S_{\epsilon}[\bar{\psi}, \psi]=\int_{\mathbf{q}, \omega} \epsilon(\mathbf{q}) \bar{\psi}(-\mathbf{q},-\omega) \psi(\mathbf{q}, \omega),
$$

where $\int_{\omega}:=\int \frac{d \omega}{2 \pi}$, and $\int_{\mathbf{q}}:=\int \frac{d^{3} q}{(2 \pi)^{3}}$ runs over the first Brillouin zone. For concreteness, we consider a cubic lattice, where the dispersion relation reads $\epsilon(\mathbf{q})=$ $4 D \sum_{\nu=1}^{3} \sin ^{2}\left(q_{\nu} a / 2\right)$ [24]. We define the time and length scale by setting the diffusion constant $D$ and the lattice spacing $a$ equal to 1 .

The mean-field rate equation follows from the "classical field equations" given by the stationarity conditions $\delta S / \delta \psi=0=\delta S / \delta \bar{\psi}$. The first equation is solved by setting the auxiliary field $\bar{\psi}=0$. Taking spatially homogeneous fields $\psi(\mathbf{x}, t) \equiv \psi(t)$ and identifying $\psi$ with the density of particles $(\psi \rightarrow \rho)$ yields the rate equation $\partial_{t} \rho=-\sum_{\mathbf{x}} \lambda(\mathbf{x}) \rho^{2}$. Hence, asymptotically the density behaves as $\rho(t) \sim\left[\sum_{\mathbf{x}} \lambda(\mathbf{x})\right]^{-1} t^{-1}$.

To account for fluctuations, one has to go beyond such a mean-field approach and consider the generating functional $W[\bar{J}, J]=\ln Z[\bar{J}, J]$, where $Z[\bar{J}, J]$ is obtained as a path integral of $\exp (-S[\bar{\phi}, \phi]+\bar{J} \bar{\phi}+J \phi)$ with respect to the fields $\bar{\phi}$ and $\phi$. This allows us to obtain an exact equation of motion for the density $\rho$ from the effective action $\Gamma[\bar{\psi}, \psi]$, the Legendre transform of $W[\bar{J}, J]$, with the stationarity conditions

$$
\delta \Gamma / \delta \psi=0=\delta \Gamma / \delta \bar{\psi} \quad \text { at } \quad \bar{\psi}=0, \quad \psi=\rho,
$$

the macroscopic analog of the classical field equations. Our analysis below will show that for the coagulation process the effective action $\Gamma$ takes a similar form as the microscopic action $S$. In fact, all but the term characterizing the coagulation process, $S_{\lambda}$, remain unaffected by fluctuations. For the latter, fluctuations renormalize the microscopic reaction kernel $\lambda(\mathbf{x})$ to its macroscopic counterpart $\mu(\mathbf{x})$. Moreover, fluctuations give rise to further contributions in the expansion of the effective action $\Gamma$, the most relevant of which is found to be proportional to $\int d t \sum_{\mathbf{x}} \bar{\psi}(\mathbf{x}, t) \psi^{5 / 2}(\mathbf{x}, t)$. Thus, to the order treated in this Letter, the "extremal principle", Eq. (1), yields the kinetic equation

$$
\partial_{t} \rho=-F(\rho),
$$

for states homogeneous in space. The leading term of the nonequilibrium force $F(\rho)$ is the LMA term $\mu \rho^{2}$, where $\mu:=\sum_{\mathbf{x}} \mu(\mathbf{x})$ is the macroscopic decay rate. In addition, there are higher order terms in the density which violate the LMA, in particular, a contribution $\sim \rho^{5 / 2}$, which is derived below.

The effective action can be calculated upon employing a nonperturbative RG analysis based on the Wetterich flow equation [16-19]

$$
\partial_{k} \Gamma_{k}[\bar{\psi}, \psi]=\frac{1}{2} \operatorname{Tr}\left\{\partial_{k} \hat{R}_{k}\left(\hat{\Gamma}_{k}^{(2)}[\bar{\psi}, \psi]+\hat{R}_{k}\right)^{-1}\right\},
$$

with the flow parameter $k$. The equation connects the microscopic action $S[\bar{\psi}, \psi] \equiv \Gamma_{k=\infty}[\bar{\psi}, \psi]$ with the macroscopic, effective action $\Gamma[\bar{\psi}, \psi] \equiv \Gamma_{k=0}[\bar{\psi}, \psi]$, where all modes are integrated. This is mediated by the cutoff term $\hat{R}_{k}$ suppressing modes with momentum $q^{2}<k^{2}$ while not affecting those with $q^{2}>k^{2}$. The trace Tr runs over the function space of $\bar{\psi}$ and $\psi . \hat{\Gamma}_{k}^{(2)}$ and $\hat{R}_{k}$ denote the $2 \times 2$ matrices of the second functional derivatives of $\Gamma_{k}$ and of the mass term $\Delta S_{k}=\int_{\mathbf{q}, \omega} R_{k}\left(q^{2}\right) \bar{\psi}(-\mathbf{q},-\omega) \psi(\mathbf{q}, \omega)$, respectively.

We first apply the RG procedure to calculate the classical LMA term, before discussing deviations from that law. To this end, we take as an ansatz a "minimal" truncation for the effective average action:

$$
\Gamma_{k}[\bar{\psi}, \psi]=S_{\lambda_{k}}[\bar{\psi}, \psi]+S_{\epsilon_{k}}[\bar{\psi}, \psi]+S_{Z_{k}}[\bar{\psi}, \psi],
$$

which contains only terms already present in the initial action. Here $S_{Z_{k}}[\bar{\psi}, \psi]=Z_{k} \int d t \sum_{\mathbf{x}} \bar{\psi} \partial_{t} \psi, \lambda_{k}$ is the renormalized reaction kernel, and $\epsilon_{k}$ is the renormalized dispersion relation. Though the RG flow generates higher order terms outside the functional space defined by the minimally truncated effective action, the flow of $\epsilon_{k}, Z_{k}$, and, under certain conditions, also $\lambda_{k}$ remains unaffected. This can be shown upon recasting the Wetterich equation in a form amenable to a diagrammatic analysis: $\partial_{k} \Gamma_{k}=$ $\tilde{\partial}_{k} \mathcal{D}_{k}$, where $\mathcal{D}_{k}=\frac{1}{2} \operatorname{Tr} \ln \left(\hat{\Gamma}_{k}^{(2)}+\hat{R}_{k}\right)$ and $\tilde{\partial}_{k}$ acts on the $k$ dependence of $\hat{R}_{k}$ only. Familiar from perturbation theory, $\mathcal{D}_{k}$ creates the one-loop Feynman diagrams to the $(m, n)$-vertex functions $\Gamma_{k}^{(m, n)}$ with propagator $1 /\left(\hat{\Gamma}_{k}^{(2)}+\right.$ $\hat{R}_{k}$ ). This flow-equation approach to RG must be integrated with the full vertex functions and propagators, which reconstructs all loop corrections.

For coagulation processes, the number of legs in the Feynman diagrams can only decrease as time passes. Hence, certain processes, e.g., corresponding to the diagram shown in Fig. 1(a), are physically not allowed, leading to a drastic restriction in possible diagrams. As a consequence, similar to the absence of propagator renormalization in perturbative RG [9], the dispersion relation and the field amplitude are not renormalized: $\epsilon_{k}(\mathbf{q})=\epsilon(\mathbf{q})$ and $Z_{k}=1$. The diagrams depicted in Figs. 1(b) and 1(c) determine the renormalization of the $\lambda_{k} \bar{\psi} \psi^{2}$ and $\lambda_{k} \bar{\psi}^{2} \psi^{2}$ term, respectively, characterizing the coagulation process. As external legs do not contribute and the internal momenta and frequencies are independent of the external ones, the diagrams give equal contributions and $\lambda_{k}$ is well-defined. Moreover, since the diagram [Fig. 1(c)] for the renormalization of the $(2,2)$-vertex function involves only $(2,2)$ vertices, one obtains a closed, analytic solution for $\lambda_{k}$. Taking the cutoff mass $R_{k}(\mathbf{q})=\left(k^{2}-\epsilon(\mathbf{q})\right) \Theta\left(k^{2}-\right.$ $\epsilon(\mathbf{q}))$ [24] yields the flow equation for the reaction kernel 
(a)

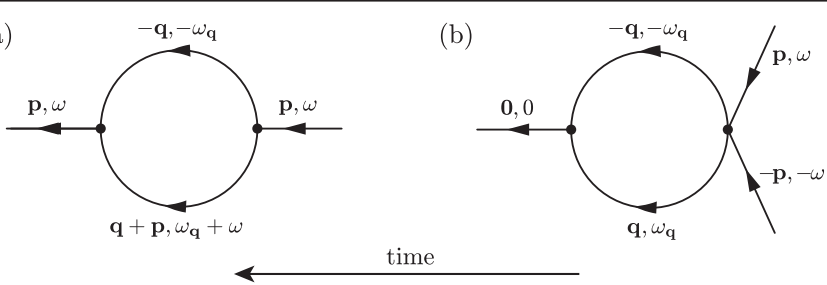

(c)

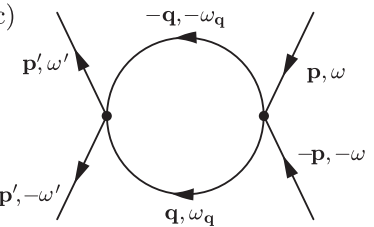

(d)

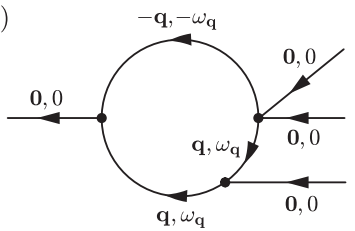

FIG. 1. Important one-loop Feynman diagrams. Because of causality, the propagator only connects earlier $\bar{\psi}$ ("creation") to later $\psi$ ("annihilation"). Since for coagulation processes the number of legs can only decrease as time passes, the set of possible diagrams is substantially restricted. Indeed, there is no such diagram as in (a) and, therefore, no renormalization of the dispersion $\epsilon$ and of the field amplitude $Z$. The diagrams depicted in (b) and (c) both stand for the renormalization of the reaction kernel $\lambda_{k}$. Finally, diagram (d) exemplifies the divergence of the RG flow contribution of one-loop diagrams which contain only $(2,1)$ and $(2,2)$ vertices: Each of the three propagators gives rise to a factor $\sim 1 / k^{2}$. For small $k$, the volume of reciprocal space and time that is integrated is $\sim k^{5}$. Thus, $g_{k}^{(1,3)} \sim k^{-1}$.

$$
\partial_{k} \lambda_{k}(\mathbf{x})=\frac{2 \lambda_{k}(\mathbf{x})\left(\mathcal{P} \circ \lambda_{k}\right)(\mathbf{x})}{k^{3}},
$$

with the projection $\left(\mathcal{P} \circ \lambda_{k}\right)(\mathbf{x})=\int_{\mathbf{q}} \exp (i \mathbf{q} \cdot \mathbf{x}) \lambda_{k}(\mathbf{q}) \cdot$ $\Theta\left(k^{2}-\epsilon(\mathbf{q})\right)$. For many reaction kernels, this simple equation is exact and allows us to analyze general coagulation processes by calculating the macroscopic decay rate $\mu=$ $\sum_{\mathbf{x}} \lambda_{k=0}(\mathbf{x})$. In particular, this applies to spheres in continuum space and particles covering only a single site on a lattice ("one-site objects"). This and the derivation of Eq. (4) are detailed in [23]. Actually, it is possible to derive a slightly more complex formula, which is exact for all reaction kernels [25].

For one-site objects, with local interactions, the flow equation reduces to $\partial_{k} \lambda_{k}=2 \lambda_{k}^{2} \int_{\mathbf{q}} \Theta\left(k^{2}-\epsilon(\mathbf{q})\right) / k^{3}$, with $\lambda_{k}:=\lambda_{k}(\mathbf{x}=\mathbf{0})$. We thus obtain an exact relation

$$
\frac{1}{\mu}=\frac{1}{\lambda_{0}}=\frac{1}{\lambda}+\int_{\mathbf{q}} \frac{1}{\epsilon(\mathbf{q})},
$$

that connects the microscopic decay rate $\lambda$ with its macroscopic analog $\mu$ by a term that depends on the lattice structure via the dispersion relation $\epsilon$. Numerical integration yields $\mu^{-1}=\lambda^{-1}+0.252731009858(3)$, in excellent agreement with our stochastic simulations; cf. Fig. 2. Equation (5) is valid not only for the cubic lattice, but for all Bravais lattices by inserting the corresponding dispersion relation. By the same token, one can treat anisotropic diffusion and arbitrary dimension $d>d_{c}$.
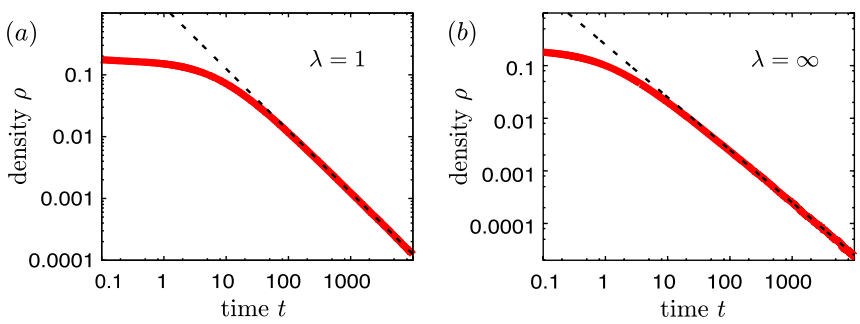

FIG. 2 (color online). Relaxation of the density for one-site objects. On double logarithmic plots, data of stochastic simulations (solid red line) are compared to the theoretical prediction for the density decay $\rho(t) \sim \mu^{-1} t^{-1}$ (dashed line), with the macroscopic decay rate $\mu^{-1}=\lambda^{-1}+0.253 \ldots$; cf. Eq. (5). The initial states in both plots were randomly distributed with $\rho(0)=0.2$.

The flow equation, Eq. (4), for the reaction kernel can also be applied to study the reaction kinetics of objects in continuous space, by simply considering the limit where the lattice spacing goes to zero. This allows us to verify Smoluchowski's result, who studied spheres that coagulate instantaneously. Indeed, as detailed in [23], we recover his result $\mu=4 \pi R$ [1], with $R$ the radius of the reaction kernel. To further illustrate the potential and versatility of our approach, we have solved Eq. (4) numerically for spheroids of equal volume $\frac{4}{3} \pi$; cf. Fig. 3 . We find that the largest values of the macroscopic rates $\lambda_{0}(\mathbf{x})$ are attained at the sharp ends and edges of the prolates and oblates, respectively. This can be traced back to the fact that large momenta $q^{2}>k^{2}$ do not contribute to the projection $\mathcal{P}$.

Finally, we extend our results beyond the lowest order in the density and discuss deviations from the LMA. To this end, we represent the nonequilibrium force as a power series

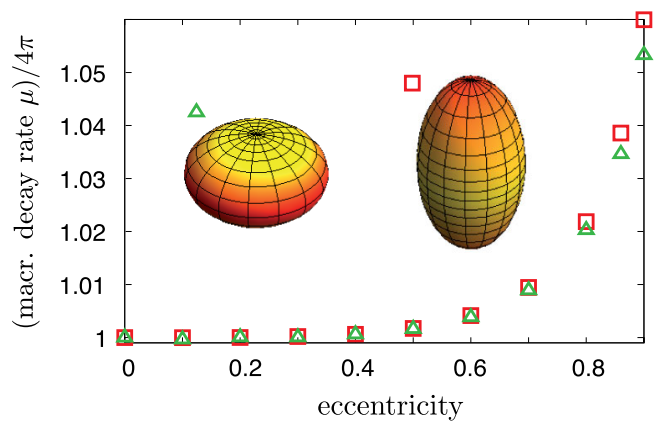

FIG. 3 (color online). Numerical solution to the flow equation, Eq. (4), for spheroids of volume $\frac{4}{3} \pi$ as a function of their eccentricity. We observe that the macroscopic decay rate $\mu$ increases with the eccentricity and is larger for prolates. In the images, as yellow (light gray) turns to orange and red (dark gray), the $\mathbf{x}$-dependent macroscopic rate $\lambda_{0}(\mathbf{x})$ grows. As a rule of thumb, the more jagged the reaction kernel, the higher $\mu$ becomes. 


$$
F(\rho)=\lim _{k \rightarrow 0} \sum_{n \geq 2} g_{k}^{(1, n)} \rho^{n},
$$

exploiting the fact that the effective average action $\Gamma_{k}$ is analytic if $k>0$ [16]. For small densities we recover $F(\rho)=\mu \rho^{2}$ with the macroscopic decay rate $\mu=g_{0}^{(1,2)}$. The flow of the coefficients $g_{k}^{(1, n)}$ is determined by diagrams with $n$ incoming and one outgoing leg. Surprisingly, the most relevant term beyond $g_{0}^{(1,2)} \rho^{2}=\mu \rho^{2}$ is not $g_{0}^{(1,3)} \rho^{3}$ as one might naively expect. In fact, in three dimensions all coefficients $g_{k}^{(1, n)}(n>2)$ turn out to diverge as $g_{k}^{(1, n)} \sim k^{5-2 n}$ for $k \rightarrow 0$. [This follows from power counting, as illustrated in Fig. 1(d).] Therefore, the infinite sum of diverging terms must scale as

$$
\sum_{n \geq 3} g_{k}^{(1, n)} \rho^{n} \sim k^{5} f\left(\frac{\rho}{k^{2}}\right),
$$

for some scaling function $f$. Since for large systems the nonequilibrium force must become independent of the system size, i.e., independent of $1 / k$, one obtains $f(x) \sim$ $x^{5 / 2}$. This adds a nonanalytic term $\sim \rho^{5 / 2}$ to the nonequilibrium force $F$.

The divergent terms in the nonequilibrium force, originating in long-wavelength fluctuations, cannot resolve the reaction kernels and the lattice structure. As elaborated in [23], this can be exploited to calculate the $\rho^{5 / 2}$ term exactly from the Wetterich equation. Overall, we find for the nonequilibrium force (exact up to higher orders in $\rho$ )

$$
F(\rho)=\mu \rho^{2}+\frac{\mu^{5 / 2}}{2 \sqrt{2} \pi} \rho^{5 / 2} .
$$

This equation bears a new fundamental insight: Beyond the LMA term, quadratic in the density, the nonequilibrium force driving the reaction kinetics contains a nonanalytic term violating the LMA. Similar as for critical phenomena, long-wavelength fluctuations are the physical origin of this term. Unlike in critical dynamics, the anomalous power law is not governed by an RG flow close to a fixed point but is a genuine strong coupling result. In contrast to lowdimensional systems, the three-dimensional coagulation process is not critical. Nevertheless, we find that the term violating the LMA is a universal function of the macroscopic decay rate $\mu$. From our theoretical analysis, we anticipate this to be a generic feature of reaction processes in three dimensions with upper critical dimension $d_{c}=2$. We have run simulations for a range of models (one-site objects with both finite and infinitely large reaction rates and two examples of extended objects that react immediately on contact) - cf. Fig. 4-which clearly corroborate our theoretical findings.

Exciton luminescence has been previously used to investigate low-dimensional reaction kinetics: By accurate measurements on the fusion of excitons, anomalous behavior was observed in an effectively one-dimensional system

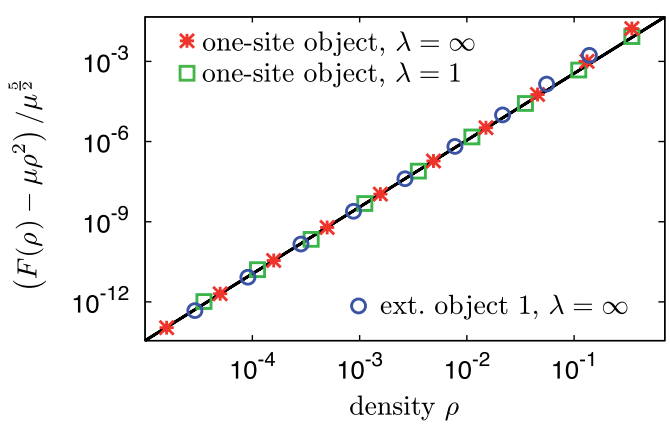

FIG. 4 (color online). Rescaled data for the deviation from the LMA. We ran simulations for a range of models and compared the results with the predicted universal correction $\rho^{5 / 2} /(2 \sqrt{2} \pi)$ (solid black line); cf. Eq. (6). The nonequilibrium force $F$ can be determined directly by introducing homogeneous particle input with rate $J$. This gives rise to an additional term $J$ in the kinetic equation, such that for the stationary state $F(\rho)=J$. Extended object 1 is a discretization of the sphere, made up of seven sites. Together with extended object 2, it is discussed in [23], where also the data for extended object 2 are provided (not shown here for clarity but in excellent agreement with the theory as well).

$[26,27]$. We expect that our prediction of a strong violation of the LMA could be revealed with similar kinds of experiments for three-dimensional systems. In addition, we believe that our results will stimulate further theoretical and experimental activities to explore the fundamental implications of fluctuations on reaction kinetics and to map out the range of validity of the LMA.

Financial support of Deutsche Forschungsgemeinschaft through the German Excellence Initiative via the program "Nanosystems Initiative Munich" (NIM) and through the SFB TR12 "Symmetries and Universalities in Mesoscopic Systems" is gratefully acknowledged.

[1] M. Smoluchowski, Z. Phys. Chem. 92, 129 (1917).

[2] S. Chandrasekhar, Rev. Mod. Phys. 15, 1 (1943).

[3] D. Toussaint and F. Wilczek, J. Chem. Phys. 78, 2642 (1983).

[4] K. Kang and S. Redner, Phys. Rev. Lett. 52, 955 (1984).

[5] K. Kang and S. Redner, Phys. Rev. A 30, 2833 (1984).

[6] R. Kopelman, Science 241, 1620 (1988).

[7] V. Kuzovkov and E. Kotomin, Rep. Prog. Phys. 51, 1479 (1988).

[8] D. ben-Avraham, Phys. Rev. Lett. 81, 4756 (1998).

[9] L. Peliti, J. Phys. A 19, L365 (1986).

[10] B. Lee, J. Phys. A 27, 2633 (1994).

[11] R. Zsigmondy, Z. Phys. Chem. 92, 600 (1917).

[12] P. Avakian and R. Merrifield, Mol. Cryst. Liq. Cryst. 5, 37 (1968).

[13] H. Zhou, Q. Rev. Biol. 43, 219 (2010).

[14] M. Doi, J. Phys. A 9, 1479 (1976).

[15] A. S. Mikhailov, Phys. Lett. 85A, 214 (1981); 85A, 427 (1981); A. M. Gutin, A.S. Mikhailov, and V. V. Yashin, Sov. Phys. JETP 65, 533 (1987). 
[16] J. Berges, N. Tetradis, and C. Wetterich, Phys. Rep. 363, 223 (2002).

[17] L. Canet, Ann. Phys. (Paris) 29, 33 (2004).

[18] L. Canet, B. Delamotte, O. Deloubrière, and N. Wschebor, Phys. Rev. Lett. 92, 195703 (2004).

[19] L. Canet, J. Phys. A 39, 7901 (2006).

[20] M. Doi, J. Phys. A 9, 1465 (1976).

[21] Ya. B. Zel'dovich and A. A. Ovchinnikov, Sov. Phys. JETP 47, 829 (1978).

[22] L. Peliti, J. Phys. (Paris) 46, 1469 (1985).
[23] See Supplemental Material at http://link.aps.org/ supplemental/10.1103/PhysRevLett.108.108301 for details.

[24] N. Dupuis and K. Sengupta, Eur. Phys. J. B 66, 271 (2008).

[25] A. A. Winkler and E. Frey (to be published).

[26] R. Blakley, C. Martinez, M. Herman, and G. McPherson, Chem. Phys. 146, 373 (1990).

[27] R. Kroon, H. Fleurent, and R. Sprik, Phys. Rev. E 47, 2462 (1993). 